\title{
Axial charges of the proton within an extended chiral constituent quark model
}

\author{
Jin-Bao Wang $\odot,{ }^{1}$ Gang $\mathrm{Li}^{2,}{ }^{2 *}$ Chun-Sheng An, ${ }^{1, \dagger}$ and Ju-Jun $\mathrm{Xie}^{3,4,5, *}$ \\ ${ }^{1}$ School of Physical Science and Technology, Southwest University, Chongqing 400715, China \\ ${ }^{2}$ College of Physics and Engineering, Qufu Normal University, Qufu 273165, China \\ ${ }^{3}$ Institute of Modern Physics, Chinese Academy of Sciences, Lanzhou 730000, China \\ ${ }^{4}$ School of Nuclear Science and Technology, University of Chinese Academy of Sciences, \\ Beijing 101408, China \\ ${ }^{5}$ School of Physics and Microelectronics, Zhengzhou University, Zhengzhou, Henan 450001, China
}

(Received 20 April 2021; accepted 1 June 2021; published 22 June 2021)

\begin{abstract}
We have performed a study of the isovector, octet, and singlet axial charges of the proton in an extended chiral constituent quark model, where all the possible $u u d q \bar{q}(q=u, d, s)$ five-quark Fock components in the proton wave function are taken into account. The ${ }^{3} P_{0}$ quark-antiquark creation mechanism is assumed to account for the transition coupling between three- and five-quark components in proton, and the corresponding transition coupling strength is fixed by fitting the intrinsic sea flavor asymmetry $\bar{d}-\bar{u}$ data for proton. Accordingly, with all the parameters fixed by empirical values, the probabilities of the intrinsic five-quark Fock components in proton wave function should be $\sim 30-50 \%$, which lead to the numerical results for quark spin $\Delta u, \Delta d$, and $\Delta s$, as well the axial charges of proton consistent with the experimental data and predictions by other theoretical approaches.
\end{abstract}

DOI: 10.1103/PhysRevD.103.114018

\section{INTRODUCTION}

Study of the structure of nucleon and nucleon excitations is one of the most important topic in hadronic physics. Experimentally, most of the explicit information about proton structure is from the deep inelastic scattering measurements. Two renowned measurements performed by European Muon Collaboration (EMC) [1,2] and New Muon Collaboration (NMC) [3], showed us that, in addition to the up and down quarks, there are also other contributions to the proton spin. Furthermore, the anti-up and antidown quarks are asymmetry inside the proton. Later, there have been also other experimental efforts dedicated to the measurements of the proton structure [3-9]. Very recently, new experimental results by SeaQuest Collaboration confirmed precisely that the distributions of anti-up and antidown quarks inside proton are considerably different and there are more antidown quark than anti-up quark over a wide range of momenta [10].

\footnotetext{
*gli@qfnu.edu.cn ancs@swu.edu.cn

*xiejujun@impcas.ac.cn
}

Published by the American Physical Society under the terms of the Creative Commons Attribution 4.0 International license. Further distribution of this work must maintain attribution to the author(s) and the published article's title, journal citation, and DOI. Funded by SCOAP.
On the other hand, one can also use the spin dependent structure function $g_{1}(x)$ of the proton to investigate the contribution of the spin of the quarks to the proton spin. It also relates the integral over all $x$ of the difference of $g_{1}(x)$ for the proton to the scale-invariant axial charges $g_{A}$ of the target proton. Indeed, in Ref. [2,11], it was shown that the flavor-singlet, isovector, and $\mathrm{SU}(3)$ octet axial charges of proton, can be obtained by combining the deep inelastic scattering (DIS) data with the nucleon and hyperon $\beta$-decay data, which are: $g_{A}^{(0)}=0.120 \pm 0.093 \pm 0.138, g_{A}^{(3)}=$ $1.254 \pm 0.006, g_{A}^{(8)}=0.688 \pm 0.035$. However, these values are obviously different with the constituent quark model predictions which are $g_{A}^{(0)}=1, g_{A}^{(3)}=5 / 3$, and $g_{A}^{(8)}=1$, respectively. In addition, the recent measurements performed by COMPASS collaboration [12,13] showed that $g_{A}^{(0)}=0.33 \pm 0.03, \quad g_{A}^{(3)}=1.2670 \pm 0.0035$, and $g_{A}^{(8)}=0.58 \pm 0.03 \pm 0.05$. Detailed reviews about the experimental and theoretical progress on proton spin puzzle and the intrinsic sea flavor asymmetry in proton, were made in Refs. [14-20]. The fact that the quark-quark interaction is relatively weak at large energy and momentum scales, whereas it is clouded by the increasingly strong interaction at lower energy scales is the reason why, so far, we have no clear picture for the structure of proton.

In fact, one has to go beyond the original three-quark $q q q$ picture of proton to explain the experimental 
measurements mentioned above. In Ji's sum rule [21], the proton spin should be decomposed as the inner intrinsic quark spin, orbital angular momentum, as well as those of the gluon inside proton. All these decomposed contents have been intensively calculated by Lattice QCD [22-27]. Phenomenologically, both the proton spin puzzle and the intrinsic sea flavor asymmetry could be solved within the meson cloud model [28,29]. It's also shown that the strangeness spin, strangeness magnetic moment, axial form factors of nucleon [30-33], and electromagnetic and strong decays of several nucleon resonances [34-37] could be described well by considering five-quark Fock components in the baryons' wave functions. In Ref. [38], the intrinsic sea content of proton was investigated by an extension of the traditional chiral constituent quark model to including the five-quark components, where the ${ }^{3} P_{0}$ model is adopted to account for the quark-antiquark pair creation in hadrons, which could result in the transitions between three- and five-quark components. Recently, the model of Ref. [38] was phenomenologically applied to study the quark orbital angular momentum in proton [39], where the theoretical calculations showed that $L_{q}=0.158 \pm 0.014$. It was shown that the study of the intrinsic sea quark content of proton is of great interest to explore its properties. Consequently, by explicit considering the contributions of the intrinsic fivequark Fock components, we employ the extended chiral constituent quark model (E $\chi \mathrm{CQM})$ of Ref. [38] to calculate the isovector, flavor-octet, and -singlet axial charges of nucleon in present work.

The present manuscript is organized as follows. In Sec. II, we give the framework which includes the extended chiral constituent quark model and the formalism for the nucleon axial charges in corresponding model, the explicit numerical results are presented in Sec. III. Finally, a brief summary is given in Sec. IV.

\section{FRAMEWORK}

In this section, we will briefly introduce the $\mathrm{E} \chi \mathrm{CQM}$ in Sec. II A, and present the formalism for calculations of the proton axial charges within present model in Sec. II B.

\section{A. $\mathbf{E} \chi \mathbf{C Q M}$}

Following Ref. [38], within the E $\chi \mathrm{CQM}$ for the ground state of octet baryons, the wave function for proton can be expressed as:

$$
|p\rangle=\frac{1}{\sqrt{\mathcal{N}}}\left(|u u d\rangle+\sum_{i} C_{i}^{q}|u u d q \bar{q}, i\rangle\right)
$$

where the first term represents the wave function for the three-quark uud component of the proton, while the sum over $i$ runs over all the possible five-quark configurations

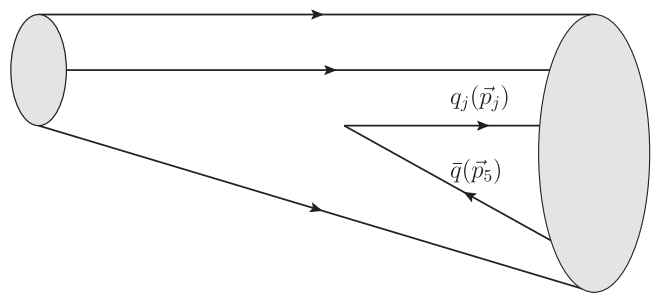

FIG. 1. Transition $q q q \rightarrow q q q q \bar{q}$ caused by a quark-antiquark pair creation in a baryon via the ${ }^{3} P_{0}$ mechanism.

with a $q \bar{q}(d \bar{d}, u \bar{u}, s \bar{s}, \ldots)^{1}$ pair which may form higher Fock components in the proton, $C_{i}^{q} / \sqrt{\mathcal{N}}$ are just the corresponding probability amplitudes for the five-quark components.

One should notice that the orbital quantum number of the inner quark and antiquark of the five-quark components in proton must be odd number $2 n+1$ because of the positive parity, while there is no obvious limit for the radial quantum number of the five-quark system.

Once an explicit quark-antiquark pair creation mechanism in the proton is pinned down, the coefficients $C_{i}^{q}$ can be calculated by

$$
C_{i}^{q}=\frac{\langle u u d q \bar{q}, i|\hat{T}| u u d\rangle}{M_{p}-E_{i}},
$$

where $M_{p}$ is the physical mass of proton, and $E_{i}$ is the energy of the $i$ th $u u d q \bar{q}$ five-quark component. The transition coupling operator $\hat{T}$ related to the quark-antiquark creation mechanism, which is taken to be the widely accepted ${ }^{3} P_{0}$ coupling mechanism in the $\mathrm{E} \chi \mathrm{CQM}$, as shown in Fig. 1, can be expressed as

$$
\begin{aligned}
\hat{T}= & -\gamma \sum_{j=1,4} \mathcal{F}_{j, 5}^{00} \mathcal{C}_{j, 5}^{00} \mathcal{C}_{\mathrm{OFSC}} \sum_{m}\langle 1, m ; 1,-m \mid 00\rangle \\
& \times \chi_{j, 5}^{1, m} \mathcal{Y}_{j, 5}^{1,-m}\left(\vec{p}_{j}-\vec{p}_{5}\right) b^{\dagger}\left(\vec{p}_{j}\right) d^{\dagger}\left(\vec{p}_{5}\right),
\end{aligned}
$$

where $\gamma$ is an dimensionless transition coupling constant for uud $\rightarrow$ uudq $\bar{q}, \mathcal{F}_{j, 5}^{00}$ and $\mathcal{C}_{j, 5}^{00}$ are the flavor and color singlet of the created quark-antiquark pair $q_{j} \bar{q}_{5}, \chi_{j, 5}^{1, m}$ and $\mathcal{Y}_{j, 5}^{1,-m}$ are the total spin $S=1$ and relative orbital $P$ - states of the created quark-antiquark system, the operator $\mathcal{C}_{\mathrm{OFSC}}$ is to calculate the overlap factor between the residual three-quark configuration in the five-quark component and the valence three-quark component, finally, $b^{\dagger}\left(\vec{p}_{j}\right), d^{\dagger}\left(\vec{p}_{5}\right)$ are the quark and antiquark creation operators.

Explicit calculations of the matrix elements of the operator $\mathcal{C}_{O F S C}$, show that the transition coupling between a $q q q q \bar{q}$ configuration with orbital quantum number $l \geq 3$

\footnotetext{
${ }^{1}$ In this work, we do not take $c \bar{c}$ and $b \bar{b}$ into account, since the probabilities for them are much smaller than other five-quark components inside the proton in the low energy scale.
} 
TABLE I. The orbital-flavor-spin configurations for five-quark configurations those may exist as higher Fock components in proton.

\begin{tabular}{|c|c|c|c|c|c|}
\hline$i$ & 1 & 2 & 3 & 4 & 5 \\
\hline Configuration & {$[31]^{\chi}[4]^{F S}[22]^{F}[22]^{S}$} & {$[31]^{\chi}[31]^{F S}[211]^{F}[22]^{S}$} & {$[31]^{\chi}[31]^{F S}[31]^{F_{1}}[22]^{S}$} & {$[31]^{\chi}[31]^{F S}[31]^{F_{2}}[22]^{S}$} & {$[4]^{\chi}[31]^{F S}[211]^{F}[22]^{S}$} \\
\hline$i$ & 6 & 7 & 8 & 9 & 10 \\
\hline Configuration & {$[4]^{\chi}[31]^{F S}[31]^{F_{1}}[22]^{S}$} & {$[4]^{\chi}[31]^{F S}[31]^{F_{2}}[22]^{S}$} & {$[31]^{\chi}[4]^{F S}[31]^{F_{1}}[31]^{S}$} & {$[31]^{\chi}[4]^{F S}[31]^{F_{2}}[31]^{S}$} & {$[31]^{\chi}[31]^{F S}[211]^{F}[31]^{S}$} \\
\hline$i$ & 11 & 12 & 13 & 14 & 15 \\
\hline Configuration & {$[31]^{\chi}[31]^{F S}[22]^{F}[31]^{S}$} & {$[31]^{\chi}[31]^{F S}[31]^{F_{1}}[31]^{S}$} & {$[31]^{\chi}[31]^{F S}[31]^{F_{2}}[31]^{S}$} & {$[4]^{\chi}[31]^{F S}[211]^{F}[31]^{S}$} & {$[4]^{\chi}[31]^{F S}[22]^{F}[31]^{S}$} \\
\hline$t$ & 16 & 17 & & & \\
\hline Configuration & {$[4]^{\chi}[31]^{F S}[31]^{F_{1}}[31]^{S}$} & {$[4]^{\chi}[31]^{F S}[31]^{F_{2}}[31]^{S}$} & & & \\
\hline
\end{tabular}

or the radial quantum number $n_{r} \neq 0$ and the $q q q$ component in proton vanishes. Consequently, there should be 17 different flavor-spin-orbital five-quark configurations which may form Fock components in the wave function of proton, which are shown in Table I. In each five-quark configuration, $[v]^{F}$ denotes the flavor wave function of fourquark subsystem, and there may be four different flavor symmetry, i.e., $[v]^{F}=[31]^{F_{1}},[31]^{F_{2}},[22]^{F}$ and $[211]^{F}$, respectively.

For the $u u d q \bar{q}$ configurations with $[v]^{F}=[31]^{F_{1}}$, the quark-antiquark pair can only be $u \bar{u}$ and $d \bar{d}$. On the other hand, one can easily obtain that the decomposition between the flavor wave function for the four-quark subsystem and the antiquark should be

$$
\left|\frac{1}{2}, \frac{1}{2}\right\rangle_{I}^{[31]^{F_{1}}}=\sqrt{\frac{2}{3}}\left|u^{3} d_{[31]^{F_{1}}}\right\rangle \otimes|\bar{u}\rangle+\sqrt{\frac{1}{3}}\left|u^{2} d_{[31]^{F_{1}}}^{2}\right\rangle \otimes|\bar{d}\rangle,
$$

where $\left|\frac{1}{2}, \frac{1}{2}\right\rangle_{I}^{[31]^{F_{1}}}$ denotes the isospin state of the proton.

For the $u u d q \bar{q}$ configurations with $[v]^{F}=[31]^{F_{2}}$, the quark-antiquark pair can only be $s \bar{s}$, and the corresponding isospin wave function of the proton is

$$
\left|\frac{1}{2}, \frac{1}{2}\right\rangle_{I}^{[31]^{F_{2}}}=\left|u^{2} d s_{[31]^{F_{2}}}\right\rangle \otimes|\bar{s}\rangle
$$

For the $u u d q \bar{q}$ configurations with $[v]^{F}=[22]^{F}$,

$$
\begin{aligned}
& \left|\frac{1}{2}, \frac{1}{2}\right\rangle_{I}^{[22]^{F}}=\left|u^{2} d_{[22]^{F}}^{2}\right\rangle \otimes|\bar{d}\rangle, \\
& \left|\frac{1}{2}, \frac{1}{2}\right\rangle_{I}^{[22]^{F}}=\left|u^{2} d s_{[22]^{F}}\right\rangle \otimes|\bar{s}\rangle,
\end{aligned}
$$

which are the isospin wave functions for the five-quark components in proton with $d \bar{d}$ and $s \bar{s}$ pairs, respectively.

At last, for the $u u d q \bar{q}$ configurations with $[v]^{F}=[211]^{F}$, which limits the quark-antiquark pair to be $s \bar{s}$,

$$
\left|\frac{1}{2}, \frac{1}{2}\right\rangle_{I}^{[211]^{F}}=\left|u^{2} d s_{[211]^{F}}\right\rangle \otimes|\bar{s}\rangle
$$

The total spin for the four-quark system with spin symmetry $[22]^{S}$ should be $S=0$, therefore, a general wave function for the five-quark configurations with numbers $i=1 \ldots 7$ can be expressed as

$$
\begin{aligned}
& |p, \uparrow\rangle=\sum_{i j k l n} \sum_{a b} \sum_{m \bar{s}_{z}} C_{1, m ; \frac{1}{2}, \bar{s}_{z}, \uparrow}^{\frac{1}{2}} C_{[31]_{k}^{\left.\chi 1^{4}\right]} ;[211]_{\bar{k}}^{C}}^{\left[3 C_{[}{ }^{[31}\right]_{k}^{\chi F S} ;[F S]_{j}^{F S}} \\
& \times C_{[F]_{l}^{F} ;[22]_{n}^{S}}^{[F S]^{F S}} C_{a, b}^{\left[2^{3}\right]^{C}}\left|[211]_{\frac{k}{C}}^{C}(a)\right\rangle\left|[11]^{C, \bar{q}}(b)\right\rangle\left|\frac{1}{2}, \frac{1}{2}\right\rangle_{I}^{[F]_{l}^{F}} \\
& \times|1, m\rangle^{[O]_{i}^{\chi}}\left|[22]_{n}^{S}\right\rangle\left|\bar{\chi}, \bar{s}_{z}\right\rangle \phi\left(\left\{\vec{r}_{q}\right\}\right),
\end{aligned}
$$

where the coefficients $C_{[\ldots]}^{[\cdots]}$...] represent the $\mathrm{CG}$ coefficients of the $S_{4}$ permutation group, $\left|[211]_{\bar{k}}^{C}(a)\right\rangle$ and $\left|[11]^{C, \bar{q}}(b)\right\rangle$ the color wave functions for the four-quark subsystem and the antiquark, combination of which results in the color singlet $\left|\left[2^{3}\right]^{C}\right\rangle$ as

$$
\left|\left[2^{3}\right]^{C}\right\rangle=\sum_{a b} C_{a, b}^{\left[2^{3}\right]^{C}}\left|[211]_{\bar{k}}^{C}(a)\right\rangle \mid[11]^{C, \bar{q}}(b) .
$$

And the state $|1, m\rangle^{[O]_{i}^{\chi}}$, which combines to the spin of the antiquark $\left|\bar{\chi}, \bar{s}_{z}\right\rangle$ to form the proton spin state $|p, \uparrow\rangle$, can be obtained from the decomposition of the orbital angular momentum of the four-quark subsystem and the antiquark.

For the configurations with numbers $i=8 \ldots 17$, the spin symmetry $[31]^{S}$ of the four-quark subsystem results in the spin $S=1$, which leads to $J=0$ or 1 when combining the orbital angular momentum of the four-quark subsystem to the antiquark. For the $J=0$ case, which has been considered explicitly in Ref. [38], a general wave function for the 10 configurations can be expressed as

$$
\begin{aligned}
|p, \uparrow\rangle= & \sum_{i j k l n} \sum_{a b} \sum_{m s_{z}} C_{1, m ; 1, s_{z}}^{00} C_{[31]_{k}^{\chi^{F S}} ;[211]_{\bar{k}}^{C}}^{\left[1^{4}\right]} C_{[O]_{i}^{\chi} ;[F S]_{j}^{F S}}^{[31]_{k}^{\chi F S}} \\
& \times C_{[F]_{l}^{F} ;[31]_{n}^{S}}^{[F S]^{F S}} C_{a, b}^{\left[2^{3}\right]^{C}}\left|[211]_{\bar{k}}^{C}(a)\right\rangle\left|[11]^{C, \bar{q}}(b)\right\rangle\left|\frac{1}{2}, \frac{1}{2}\right\rangle_{I}^{[F]_{l}^{F}} \\
& \times|1, m\rangle^{[O]_{i}^{\chi}}\left|[31]_{n}^{S}, s_{z}\right\rangle\left|\bar{\chi}, \bar{s}_{z}\right\rangle \phi\left(\left\{\vec{r}_{q}\right\}\right) .
\end{aligned}
$$


While for the $J=1$ cases,

$$
\begin{aligned}
& |p, \uparrow\rangle=\sum_{i j k l n} \sum_{a b} \sum_{J_{z} \bar{s}_{z}} \sum_{m s_{z}} C_{1, J_{z} ; \frac{1}{2}, \bar{s}_{z}}^{\frac{1}{2}, \frac{1}{2}} C_{1, m ; 1, s_{z}}^{1, J_{z}} C_{[31]_{k}^{X F S} ;[211]_{\bar{k}}^{C}}^{\left[1^{4}\right]} \\
& \times C_{[O]_{i}^{*} ;[F S]_{j}^{F F}}^{\left[31 \chi^{F F S}\right.} C_{[F]_{l}^{F} ;[31]_{n}^{S}}^{\left[F S F^{F S}\right.} C_{a, b}^{\left[2^{3}\right]^{C}}\left|[211]_{\bar{k}}^{C}(a)\right\rangle\left|[11]^{C, \bar{q}}(b)\right\rangle \\
& \times\left|\frac{1}{2}, \frac{1}{2}\right\rangle_{I}^{[F]_{l}^{F}}|1, m\rangle^{[O]_{i}^{\chi}}\left|[31]_{n}^{S}, s_{z}\right\rangle\left|\bar{\chi}, \bar{s}_{z}\right\rangle \phi\left(\left\{\vec{r}_{q}\right\}\right) .
\end{aligned}
$$

In present work, we consider both $J=0$ and $J=1$ cases. Hereafter, we denote these two cases as Set I and Set II, respectively.

Finally, to calculate the energy for a given five-quark configuration $E_{i}$ in Eq. (2), we employ the traditional chiral constituent quark model, in which the quark-quark hyperfine interaction is [40]

$$
\begin{aligned}
H_{\text {hyp }}= & -\sum_{i<j} \vec{\sigma}_{i} \cdot \vec{\sigma}_{j}\left[\sum_{a=1}^{3} V_{\pi}\left(r_{i j}\right) \lambda_{i}^{a} \lambda_{j}^{a}\right. \\
& \left.+\sum_{a=4}^{7} V_{K}\left(r_{i j}\right) \lambda_{i}^{a} \lambda_{j}^{a}+V_{\eta}\left(r_{i j}\right) \lambda_{i}^{8} \lambda_{j}^{8}\right],
\end{aligned}
$$

where $\lambda_{i}^{a}$ is the Gell-Mann matrix in flavor space which acts on the $i$ th quark, $V_{M}\left(r_{i j}\right)$ is the potential of the meson $M$ exchange interaction between $i$ th quark and $j$ th quark. Numerical values for all the exchange coupling strength constants are taken to be the empirical ones [40]. Then the energies $E_{i}$ for the 17 five-quark configurations in Table I should be

$$
E_{i}=E_{0}+\left\langle H_{\text {hyp }}\right\rangle+\delta_{q \bar{q}},
$$

where $E_{0}$ is a degenerated energy for the 17 five-quark configurations. The parameter $E_{0}$ is dependent on the constituent quark masses, the kinetic quark energies, and also the energies of the quark confinement interactions. Here we take $E_{0}=2127 \mathrm{MeV}, \delta_{u \bar{u}}=\delta_{d \bar{d}}=0$, and $\delta_{s \bar{s}}=$ $240 \mathrm{MeV}$ as used in Ref. [38].

\section{B. Formalism for the axial charges of proton}

In this section, we present the formalism for the axial charges $g_{A}^{(0)}, g_{A}^{(8)}$, and $g_{A}^{(3)}$ of proton within the E $\chi \mathrm{CQM}$.

It is shown that quark spin contribution $\Delta q$ measured in polarized DIS is related to the matrix element of the quark axial vector current operator as

$$
\left\langle p, s_{z}\left|\int \mathrm{d} x \bar{q} \gamma^{\mu} \gamma^{5} q\right| p, s_{z}\right\rangle=s^{\mu} \cdot \Delta q,
$$

where $s^{\mu}$ is the proton polarization vector, and $\Delta q$ the difference of quark with spin parallel or antiparallel to the proton spin

$$
\Delta q=\left(q^{\uparrow}+\bar{q}^{\uparrow}\right)-\left(q^{\downarrow}+\bar{q}^{\downarrow}\right) .
$$

Combinations of different flavor $\Delta f$ with $f=u, d, s$ lead to isovector, flavor octet, and flavor singlet axial charges of proton,

$$
\begin{gathered}
g_{A}^{(3)}=\Delta u-\Delta d, \\
g_{A}^{(8)}=\Delta u+\Delta d-2 \Delta s, \\
g_{A}^{(0)}=\Delta u+\Delta d+\Delta s .
\end{gathered}
$$

In the nonrelativistic approximation, $\Delta f$ can be calculated as:

$$
\Delta f=\left\langle p s_{z}\left|\sum_{j} \hat{\sigma}_{j}^{z} \delta_{j f}\right| p s_{z}\right\rangle,
$$

where $\left|p s_{z}\right\rangle$ is the wave function Eq. (1) of proton, $\hat{\sigma}_{j}^{z}$ is Pauli operator acting on $j$ th quark, and $\delta_{j f}$ is a flavordependent operator. $\delta_{j f}=1$ when the flavor of $j$ th quark is same with $f$, otherwise, $\delta_{j f}=0$. Consequently, one can get

$$
\begin{aligned}
\Delta f= & \frac{1}{\mathcal{N}}\left\langle u u d, s_{z}\left|\sum_{j=1,3} \hat{\sigma}_{j}^{z} \delta_{j f}\right| u u d, s_{z}\right\rangle \\
& +\sum_{i} \frac{\left(C_{i}^{q}\right)^{2}}{\mathcal{N}}\left\langle u u d q \bar{q}, i, s_{z}\left|\sum_{j=1,5} \hat{\sigma}_{j}^{z} \delta_{j f}\right| u u d q \bar{q}, i, s_{z}\right\rangle,
\end{aligned}
$$

where the nondiagonal terms are assumed to be negligible.

For simplicity, we denote the matrix elements for an explicit given five-quark component in the second term in Eq. (21) as

$$
\Delta f_{i}^{q}=\left\langle u u d q \bar{q}, i, s_{z}\left|\sum_{j=1}^{5} \hat{\sigma}_{j}^{z} \delta_{j f}\right| u u d q \bar{q}, i, s_{z}\right\rangle .
$$

Finally, one can easily obtain the explicit expression of the matrix elements results for $\Delta u, \Delta d$, and $\Delta s$ of proton as following

$$
\begin{gathered}
\Delta u=\frac{1}{\mathcal{N}} \frac{4}{3}+\sum_{i, q} \frac{\left(C_{i}^{q}\right)^{2}}{\mathcal{N}} \Delta u_{i}^{q}, \\
\Delta d=\frac{1}{\mathcal{N}}\left(-\frac{1}{3}\right)+\sum_{i, q} \frac{\left(C_{i}^{q}\right)^{2}}{\mathcal{N}} \Delta d_{i}^{q}, \\
\Delta s=\sum_{i, q} \frac{\left(C_{i}^{q}\right)^{2}}{\mathcal{N}} \Delta s_{i}^{q} .
\end{gathered}
$$




\section{THE NUMERICAL RESULTS}

Using the formalism developed in the previous section, here we present our numerical results for the axial charges of the proton. Before showing the theoretical results, we first discuss the model parameter $V$, which is the coupling strength for Goldstone boson exchange. The parameter $V$ can be easily obtained from the matrix elements of the operator in Eq. (3), and it is a common factor for all the five-quark configurations listed in Table I, in which the transition coupling constante $\gamma$ is included. As discussed in Sec. II A, we have two different sets in present calculations. For Set I, we just employ the numerical values for the parameters using in Ref. [38], where $J=0$ was considered. While for Set II, we keep all the parameters to be the same values as Set I except for $V$, which is, now, for the case of $J=1$. For Set II, the value of $V$ is determined by fitting the sea flavor asymmetry of proton $\bar{d}-\bar{u}=0.118 \pm 0.012[9]$, and one can get

$$
V=697 \pm 80 \mathrm{MeV}
$$

where the uncertainty is obtained from the experimental error of the value of $\bar{d}-\bar{u}$.

Accordingly, explicit calculations result in the numerical results for the probabilities of five-quark components $P_{q \bar{q}}$, and contributions to the quark spin of each five-quark configuration $\Delta f_{i}^{q}$ shown in Table II for Set I and II, respectively. Note that we have listed the quark-antiquark

TABLE II. The numerical results of the probabilities $P_{q \bar{q}}$ and the matrix elements of the quark spin $\Delta f_{i}^{q}$ of all the 17 five-quark configurations in Set I and Set II. Note that we have denoted the five-quark configurations $u u d q \bar{q}$ with light quark-antiquark pairs as $l \bar{l}$, and those with strange quark-antiquark pairs as $s \bar{s}$.

\begin{tabular}{|c|c|c|c|c|c|c|c|c|c|}
\hline \multirow[b]{2}{*}{ Configuration $i$} & \multirow[b]{2}{*}{$q \bar{q}$} & \multicolumn{4}{|c|}{ Set I } & \multicolumn{4}{|c|}{ Set II } \\
\hline & & $P_{q \bar{q}}$ & $\Delta u_{i}^{q}$ & $\Delta d_{i}^{q}$ & $\Delta s_{i}^{q}$ & $P_{q \bar{q}}$ & $\Delta u_{i}^{q}$ & $\Delta d_{i}^{q}$ & $\Delta s_{i}^{q}$ \\
\hline \multirow[t]{2}{*}{1} & $\overline{l l}$ & $0.146 \pm 0.015$ & 0 & $-1 / 3$ & 0 & $0.157 \pm 0.016$ & 0 & $-1 / 3$ & 0 \\
\hline & $s \bar{s}$ & $0.010 \pm 0.001$ & 0 & 0 & $-1 / 3$ & $0.011 \pm 0.002$ & 0 & 0 & $-1 / 3$ \\
\hline \multirow[t]{2}{*}{2} & $\overline{l l}$ & 0 & $\cdots$ & $\cdots$ & $\cdots$ & 0 & $\cdots$ & $\cdots$ & $\cdots$ \\
\hline & $s \bar{s}$ & $0.004 \pm 0.001$ & 0 & 0 & $-1 / 3$ & $0.004 \pm 0.001$ & 0 & 0 & $-1 / 3$ \\
\hline \multirow[t]{2}{*}{3} & $\overline{l l}$ & $0.016 \pm 0.002$ & $-2 / 9$ & $-1 / 9$ & 0 & $0.018 \pm 0.002$ & $-2 / 9$ & $-1 / 9$ & 0 \\
\hline & $s \bar{s}$ & 0 & $\cdots$ & $\cdots$ & $\ldots$ & 0 & $\ldots$ & $\ldots$ & $\ldots$ \\
\hline \multirow[t]{2}{*}{4} & $\overline{l l}$ & 0 & $\cdots$ & $\cdots$ & $\cdots$ & 0 & $\cdots$ & $\cdots$ & $\cdots$ \\
\hline & $s \bar{s}$ & $0.003 \pm 0.001$ & 0 & 0 & $-1 / 3$ & $0.003 \pm 0.001$ & 0 & 0 & $-1 / 3$ \\
\hline \multirow[t]{2}{*}{5} & $\overline{l l}$ & 0 & $\cdots$ & $\cdots$ & $\ldots$ & 0 & $\cdots$ & $\cdots$ & $\cdots$ \\
\hline & $s \bar{s}$ & $0.009 \pm 0.001$ & 0 & 0 & $-1 / 3$ & $0.009 \pm 0.001$ & 0 & 0 & $-1 / 3$ \\
\hline \multirow[t]{2}{*}{6} & $\overline{l l}$ & $0.041 \pm 0.004$ & $-2 / 9$ & $-1 / 9$ & 0 & $0.045 \pm 0.005$ & $-2 / 9$ & $-1 / 9$ & 0 \\
\hline & $s \bar{s}$ & 0 & $\ldots$ & $\ldots$ & $\ldots$ & 0 & $\cdots$ & $\ldots$ & $\cdots$ \\
\hline \multirow[t]{2}{*}{7} & $\overline{l l}$ & 0 & $\cdots$ & $\cdots$ & $\cdots$ & 0 & $\cdots$ & $\cdots$ & $\cdots$ \\
\hline & $s \bar{s}$ & $0.007 \pm 0.001$ & 0 & 0 & $-1 / 3$ & $0.007 \pm 0.001$ & 0 & 0 & $-1 / 3$ \\
\hline \multirow[t]{2}{*}{8} & $\overline{l l}$ & $0.073 \pm 0.007$ & $2 / 3$ & $1 / 3$ & 0 & $0.157 \pm 0.016$ & $4 / 9$ & $-1 / 9$ & 0 \\
\hline & $s \bar{s}$ & 0 & $\ldots$ & $\cdots$ & $\cdots$ & 0 & $\cdots$ & $\cdots$ & $\ldots$ \\
\hline \multirow[t]{2}{*}{9} & $\overline{l l}$ & 0 & $\ldots$ & $\ldots$ & $\ldots$ & 0 & $\ldots$ & $\ldots$ & $\ldots$ \\
\hline & $s \bar{s}$ & $0.006 \pm 0.001$ & 0 & 0 & 1 & $0.014 \pm 0.002$ & $4 / 9$ & $-1 / 9$ & 0 \\
\hline \multirow[t]{2}{*}{10} & $\overline{l l}$ & 0 & $\cdots$ & $\cdots$ & $\cdots$ & 0 & $\cdots$ & $\cdots$ & $\cdots$ \\
\hline & $s \bar{s}$ & $0.003 \pm 0.001$ & 0 & 0 & 1 & $0.007 \pm 0.001$ & $1 / 2$ & $1 / 12$ & $-1 / 4$ \\
\hline \multirow[t]{2}{*}{11} & $\overline{l l}$ & $0.006 \pm 0.001$ & 0 & 1 & 0 & $0.013 \pm 0.001$ & $1 / 3$ & 0 & 0 \\
\hline & $s \bar{s}$ & $0.002 \pm 0.000$ & 0 & 0 & 1 & $0.004 \pm 0.001$ & $1 / 3$ & $1 / 6$ & $-1 / 6$ \\
\hline \multirow[t]{2}{*}{12} & $\overline{l l}$ & $0.005 \pm 0.001$ & $2 / 3$ & $1 / 3$ & 0 & $0.010 \pm 0.001$ & $1 / 3$ & 0 & 0 \\
\hline & $s \bar{s}$ & 0 & $\ldots$ & $\ldots$ & $\ldots$ & 0 & $\ldots$ & $\ldots$ & $\ldots$ \\
\hline \multirow[t]{2}{*}{13} & $\overline{l l}$ & 0 & $\ldots$ & $\ldots$ & $\ldots$ & 0 & $\ldots$ & $\ldots$ & $\cdots$ \\
\hline & $s \bar{s}$ & $0.001 \pm 0.000$ & 0 & 0 & 1 & $0.002 \pm 0.001$ & $7 / 18$ & $1 / 36$ & $-1 / 12$ \\
\hline \multirow[t]{2}{*}{14} & $\overline{l l}$ & 0 & $\cdots$ & $\cdots$ & $\cdots$ & 0 & $\cdots$ & $\cdots$ & $\cdots$ \\
\hline & $s \bar{s}$ & $0.008 \pm 0.001$ & 0 & 0 & 1 & $0.017 \pm 0.002$ & $1 / 2$ & $1 / 12$ & $-1 / 4$ \\
\hline \multirow[t]{2}{*}{15} & $\overline{l l}$ & $0.015 \pm 0.002$ & 0 & 1 & 0 & $0.032 \pm 0.003$ & $1 / 3$ & 0 & 0 \\
\hline & $s \bar{s}$ & $0.004 \pm 0.001$ & 0 & 0 & 1 & $0.009 \pm 0.001$ & $1 / 3$ & $1 / 6$ & $-1 / 6$ \\
\hline \multirow[t]{2}{*}{16} & $\overline{l l}$ & $0.012 \pm 0.001$ & $2 / 3$ & $1 / 3$ & 0 & $0.025 \pm 0.002$ & $1 / 3$ & 0 & 0 \\
\hline & $s \bar{s}$ & 0 & $\ldots$ & $\ldots$ & $\ldots$ & 0 & $\ldots$ & $\ldots$ & $\ldots$ \\
\hline \multirow[t]{2}{*}{17} & $\overline{l l}$ & 0 & $\cdots$ & $\cdots$ & $\cdots$ & 0 & $\cdots$ & $\cdots$ & $\cdots$ \\
\hline & $s \bar{s}$ & $0.002 \pm 0.000$ & 0 & 0 & 1 & $0.004 \pm 0.001$ & $7 / 18$ & $1 / 36$ & $-1 / 12$ \\
\hline
\end{tabular}


pairs in all the different $u u d q \bar{q}$ configurations being light $(u \bar{u}$ or $d \bar{d})$ and strange flavors in two rows denoted by $\bar{l}$ and $s \bar{s}$ in the table, respectively.

It's shown that $\Delta f_{i}^{q}$ are the same ones for the five-quark configurations with spin symmetry $[22]^{S}$ in Set I and Set II, this is because that both the two sets share the same wave function of Eq. (9) for these configurations. For configurations numbered $i=8-17$, whose wave functions are Eqs. (11) and (12) for Set I and II, respectively, the obtained $\Delta f_{i}^{q}$ are therefore different. While the probabilities for any configurations in the wave function of proton should be different for the two sets. Indeed, for the case of $J=0$, the total obtained probabilities for the five-quark components with $u \bar{u}, d \bar{d}$, and $s \bar{s}$ pairs are

$$
\begin{gathered}
P_{u \bar{u}}=0.098 \pm 0.010, \\
P_{d \bar{d}}=0.216 \pm 0.022, \\
P_{s \bar{s}}=0.057 \pm 0.006 .
\end{gathered}
$$

These numerical values yield the ratios

$$
\begin{gathered}
r_{l}=\frac{P_{u \bar{u}}}{P_{d \bar{d}}}=0.5 \pm 0.1, \\
r_{s}=\frac{P_{s \bar{s}}}{P_{d \bar{d}}}=0.3 \pm 0.1, \\
\kappa_{s}=\frac{2 P_{s \bar{s}}}{P_{u \bar{u}}+P_{d \bar{d}}}=0.4 \pm 0.1,
\end{gathered}
$$

which can be related to the ratios of pseudoscalar meson electroproduction. These ratios were extracted to be $r_{l}=$ $0.74 \pm 0.18, r_{s}=0.22 \pm 0.07$ and $0.25 \pm 0.09$ by CLAS collaboration [41]. It is found that, within errors, our results are roughly consistent with these above experimental data.

While for the case of $J=1$, the theoretical results are

$$
\begin{gathered}
P_{u \bar{u}}=0.170 \pm 0.017, \\
P_{d \bar{d}}=0.288 \pm 0.029, \\
P_{s \bar{s}}=0.090 \pm 0.009 .
\end{gathered}
$$

The probabilities for both light and strange five-quark Fock components are larger than those obtained by Set I, which is because of that the coupling strength between the five-quark configurations with the four-quark spin symmetry $[31]^{S}$ and the traditional three-quark component of proton for Set II is larger than that for Set I. Accordingly, one can get the ratios

$$
\begin{aligned}
& r_{l}=0.6 \pm 0.1, \\
& r_{s}=0.3 \pm 0.1, \\
& \kappa_{s}=0.4 \pm 0.1,
\end{aligned}
$$

these values are similar with those obtained by Set I, but one has to note that the total probability of the five-quark components in Set II is relatively large, and the numerical results are in agreement with both the $\bar{d}-\bar{u}$ and $\bar{u} / \bar{d}$ data quite well. In addition, the present obtained probabilities of the five-quark components with light quark-antiquark pair are very close to the results obtained by the BHPS model that [42]

$$
P_{u \bar{u}}=0.176, \quad P_{d \bar{d}}=0.294 .
$$

Next, we discuss in detail the theoretical results in the following two subsections for Set I and Set II, respectively.

\section{A. Set I}

From the quark spin matrix elements $\Delta f_{i}^{q}$ obtained from the wave function as in Eqs. (9) and (11) for Set I, one can easily find that the total spin and total angular momentum of the four-quark subsystem in the five-quark configurations with four-quark orbital symmetry $[31]^{\chi}$ are 0 . For the configurations with four-quark spin symmetry $[22]^{S}$ and orbital symmetry $[4]^{\chi}$, and for those with four-quark spin symmetry $[31]^{S}$ and orbital symmetry $[4]^{\chi}$, the total orbital angular momentum of the quarks and the antiquark are also 0 . Thus, for all the 17 five-quark configurations, only the antiquark spin contributes to $\Delta f_{i}^{q}$.

With the obtained probabilities $P_{q \bar{q}}$ and the matrix elements $\Delta f_{i}^{q}$, we get the total quark spin $\Delta f$ shown in Table III, and the axial charges of proton shown in Table IV, compared to the experimental measurement by COMPASS [13] in the rows denoted by EXP, and the latest predictions by lattice QCD $[13,24,27,43,44]$ in the rows denoted by LQCD, predictions by the extended cloudy bag model [45] in the row denoted by $\mathrm{CMB}$, and predictions by the chiral perturbation theory [46] in the row denoted by $\chi \mathrm{PT}$.

As one can see in Table III, predictions for $\Delta u$ by most of the theoretical approaches are about $0.8-0.9$ which fall very well in the range of the COMPASS data, while those for $\Delta d$ and $\Delta s$ show a little deviation from the data. Similarly, the present obtained $\Delta u$ is very close to the data, but $\Delta d$ is only about half of the experimental value, while the worst result is that the sign of the present $\Delta s$ is contrary to that of the experimental result, as well the predictions by other approaches. As discussed above, in present case, only the antiquark spin contributes to $\Delta f_{i}^{q}$, and the spin states of the anti-strange quark in the configurations numbered 8-17 should be $|1 / 2, \uparrow\rangle$, therefore, we obtain a small but positive value for $\Delta s$. Most of the predictions for $\Delta s$ by other theoretical approaches are roughly consistent with the COMPASS measurement, while a recent investigation employing chiral perturbation theory showed a very close to 0 but negative value $[46,47]$.

In Table IV, the obtained $g_{A}^{(3)}$ and $g_{A}^{(8)}$ are in agreement with the data and these predictions from other approaches, with a $\sim 10 \%$ deviation. But the present $g_{A}^{(0)}$, which should 
TABLE III. Numerical results of $\Delta f(f=u, d, s)$, compared to the data and predictions by lattice QCD and chiral perturbation theory.

\begin{tabular}{lccc}
\hline \hline & $\Delta u$ & $\Delta d$ & $\Delta s$ \\
\hline Set I & $0.883 \pm 0.005$ & $-0.213 \pm 0.003$ & $0.015 \pm 0.002$ \\
Set II & $0.710 \pm 0.012$ & $-0.225 \pm 0.008$ & $-0.020 \pm 0.003$ \\
EXP [13] & $0.84 \pm 0.01 \pm 0.02$ & $-0.430 \pm 0.01 \pm 0.02$ & $-0.030 \pm 0.01 \pm 0.02$ \\
LQCD [24] & $0.794(21)(2)$ & $-0.289(16)(1)$ & $-0.023(10)(1)$ \\
LQCD [43] & $0.863(7)(14)$ & $-0.345(6)(9)$ & $-0.0240(21)(11)$ \\
LQCD [27] & & & $-0.046(26)(9)$ \\
LQCD [44] & $0.864(16)$ & $-0.426(16)$ & $-0.046(8)$ \\
$\chi$ PT [46] & $0.90_{-0.04}^{+0.03}$ & $-0.38_{0.03}^{+0.03}$ & $-0.007_{-0.007}^{+0.004}$ \\
\hline \hline
\end{tabular}

TABLE IV. Numerical results of $g_{A}^{(3)}, g_{A}^{(8)}$, and $g_{A}^{(0)}$, compared to the data and predictions by extended cloudy bag model, and lattice QCD and chiral perturbation theory.

\begin{tabular}{lccc}
\hline \hline & $g_{A}^{(3)}$ & $g_{A}^{(8)}$ & $g_{A}^{(0)}$ \\
\hline Set I & $1.096 \pm 0.0053$ & $0.640 \pm 0.010$ & $0.685 \pm 0.0076$ \\
Set II & $0.935 \pm 0.0192$ & $0.525 \pm 0.0258$ & $0.465 \pm 0.0225$ \\
EXP [13] & $1.2670(35)$ & $0.58 \pm 0.03 \pm 0.05$ & $0.33 \pm 0.03$ \\
LQCD [24] & $1.082(18)(2)$ & $0.550(24)(1)$ & $0.482(38)(2)$ \\
LQCD [43] & $1.208(6)(16)(1)(10)$ & $0.565(11)(13)$ & $0.494(11)(15)$ \\
LQCD [27] & $1.123(28)(29)(90)$ & & $0.420 \pm 0.02$ \\
CBM [45] & 1.270 & $0.53_{-0.06}^{+0.06}$ & $0.370 \pm 0.02$ \\
$\chi$ PT [46] & 1.27 & & $0.51_{-0.08}^{+0.07}$ \\
\hline \hline
\end{tabular}

indicate the quark spin contributions to proton spin, is more than twice of the COMPASS data, this is because of the small absolute value of $\Delta d$ and the positive $\Delta s$ obtained in present model. In Ref. [45], a cloudy bag model was applied to the axial charges of proton, the $N \pi, \Delta \pi$, and $\Lambda K$ Fock components were taken into account, by considering the relativistic corrections, they obtained the numerical results for proton axial charges which fitted the experimental data very well.

\section{B. Set II}

For the quark spin matrix elements $\Delta f_{i}^{q}$, differing from Set I, both the quarks and antiquarks in the configurations numbered 8-17 could contribute. And with the numerical results for $P_{q \bar{q}}$ and $\Delta f_{i}^{q}$, we get the results of the quark spin $\Delta f$ and the axial charges of proton listed in Table III and IV, respectively, both results are compared to the experimental data and predictions by other theoretical approaches.

One can immediately find that the present obtained $\Delta u$ has a deviation from the experimental data by more than $10 \%$, and the numerical value for $\Delta d$ is very close to the one obtained by Set I, which is smaller than the COMPASS data. The $\Delta s$ is small and negative. All these numerical results for $\Delta f$ are close to the results published by QCDSF collaboration [24].

For the isovector, flavor octet and singlet axial charges of the proton, as one can see in Table IV, the obtained $g_{A}^{(3)}$ is only about $3 / 4$ of the COMPASS data. This is because a relatively small value of $\Delta u$ is obtained in present Set II. While numerical values of the flavor octet axial charge $g_{A}^{(8)}$ and the flavor singlet axial charge $g_{A}^{(0)}$ are very close to the lattice QCD predictions in Refs. [24,43].

\section{SUMMARY}

To summarize, in present manuscript, the intrinsic sea content in proton is investigated using an extended chiral constituent quark model in which all the five-quark Fock components are taken into account. We take the ${ }^{3} P_{0}$ quarkantiquark creation mechanism to get the transition coupling strength between the three- and five-quark components. Taking the empirical values for the model parameters, one can get the total probability of all possible five-quark components in proton wave function which is about 30-50\%. The theoretical calculations can fit both the sea flavor asymmetry $\bar{d}-\bar{u}[9]$ and the strangeness suppression of $q \bar{q}$ creation obtained recently by CLAS collaboration [41].

In present phenomenological work, to form the positive parity of proton, one of the quarks or antiquark in a given five-quark Fock component of the proton wave function must be in the orbital $P$-state, which contribute a value $\sim 0.158$ to the spin of proton [39]. Consequently, the obtained wave function for proton in present work is then applied to study the inner quark spin of proton. 
Our numerical results show that the obtained $\Delta u, \Delta d$, and $\Delta s$ are in consistent with the experimental data and predictions by other theoretical approaches.

Within the obtained proton wave functions, we study the isovector, flavor octet and singlet axial charges. It is shown that the probabilities $P_{q \bar{q}}$ might reach to $\sim 50 \%$ for the fivequark Fock components in proton, and the resulting values for $g_{A}^{(3)}, g_{A}^{(0)}$, and $g_{A}^{(8)}$ are consistent with the predictions by other theoretical approaches. Note that we have considered only the contributions from the inner quark spin, thus the present obtained numerical results are not fully in agreement with the experimental data. We will also study contributions from the gluon spin and orbital angular momentum, in future, when more experimental data are available.

\section{ACKNOWLEDGMENTS}

This work is partly supported by the National Natural Science Foundation of China under Grants No. 11675131, No. 12075288, No. 12075133, No. 11735003, No. 11961141012, and No. 11835015. It is also supported by the Youth Innovation Promotion Association CAS, Taishan Scholar Project of Shandong Province (Grant No. tsqn202103062) and the Higher Educational Youth Innovation Science and Technology Program Shandong Province (Grant No. 2020KJJ004).
[1] J. Ashman et al. (European Muon Collaboration), A measurement of the spin asymmetry and determination of the structure function $\mathrm{g}(1)$ in deep inelastic muon-the proton scattering, Phys. Lett. B 206, 364 (1988).

[2] J. Ashman et al. (European Muon Collaboration), An investigation of the spin structure of the proton in deep inelastic scattering of polarized muons on polarized protons, Nucl. Phys. B328, 1 (1989).

[3] M. Arneodo et al. (New Muon Collaboration), A reevaluation of the Gottfried sum, Phys. Rev. D 50, R1 (1994).

[4] P. Amaudruz et al. (New Muon Collaboration), The Gottfried Sum from the Ratio $F_{2}^{n} / F_{2}^{p}$, Phys. Rev. Lett. 66, 2712 (1991).

[5] P. L. McGaughey et al. (E772 Collaboration), Limit on the $\bar{d} / \bar{u}$ Asymmetry of the Nucleon Sea from Drell-Yan Production, Phys. Rev. Lett. 69, 1726 (1992).

[6] A. Baldit et al. (NA51 Collaboration), Study of the isospin symmetry breaking in the light quark sea of the nucleon from the Drell-Yan process, Phys. Lett. B 332, 244 (1994).

[7] K. Ackerstaff et al. (HERMES Collaboration), The Flavor Asymmetry of the Light Quark Sea from Semi-Inclusive Deep Inelastic Scattering, Phys. Rev. Lett. 81, 5519 (1998).

[8] P. L. Anthony et al. (E155 Collaboration), Measurements of the $Q^{2}$ dependence of the proton and neutron spin structure functions $g_{1}^{p}$ and $g_{1}^{n}$, Phys. Lett. B 493, 19 (2000).

[9] R.S. Towell et al. (FNAL E866/NuSea Collaboration), Improved measurement of the $\bar{d} / \bar{u}$ asymmetry in the nucleon sea, Phys. Rev. D 64, 052002 (2001).

[10] J. Dove et al. (SeaQuest Collaboration), The asymmetry of antimatter in the proton, Nature (London) 590, 561 (2021).

[11] C. A. Aidala, S. D. Bass, D. Hasch, and G. K. Mallot, The spin structure of the nucleon, Rev. Mod. Phys. 85, 655 (2013).

[12] V. Y. Alexakhin et al. (COMPASS Collaboration), The deuteron spin-dependent structure function $g_{1}^{d}$ and its first moment, Phys. Lett. B 647, 8 (2007).

[13] M. G. Alekseev et al. (COMPASS Collaboration), The spindependent structure function of the proton $g_{1}^{p}$ and a test of the Bjorken sum rule, Phys. Lett. B 690, 466 (2010).
[14] S. E. Kuhn, J.-P. Chen, and E. Leader, Spin structure of the nucleon-status and recent results, Prog. Part. Nucl. Phys. 63, 1 (2009).

[15] M. Burkardt, C. A. Miller, and W. D. Nowak, Spinpolarized high-energy scattering of charged leptons on nucleons, Rep. Prog. Phys. 73, 016201 (2010).

[16] W. C. Chang and J. C. Peng, Flavor structure of the nucleon sea, Prog. Part. Nucl. Phys. 79, 95 (2014).

[17] E. Leader and C. Lorcé, The angular momentum controversy: What's it all about and does it matter? Phys. Rep. 541, 163 (2014).

[18] M. Wakamatsu, Is gauge-invariant complete decomposition of the nucleon spin possible? Int. J. Mod. Phys. A 29, 1430012 (2014).

[19] K. F. Liu and C. Lorcé, The parton orbital angular momentum: Status and prospects, Eur. Phys. J. A 52, 160 (2016).

[20] A. Deur, S. J. Brodsky, and G. F. De Tramond, The spin structure of the nucleon, Rep. Prog. Phys. 82, 076201 (2019).

[21] X. D. Ji, Gauge-Invariant Decomposition of Nucleon Spin, Phys. Rev. Lett. 78, 610 (1997).

[22] S. Aoki, M. Doui, T. Hatsuda, and Y. Kuramashi, Tensor charge of the nucleon in lattice QCD, Phys. Rev. D 56, 433 (1997).

[23] P. Hagler et al. (LHPC Collaboration), Nucleon generalized parton distributions from full lattice QCD, Phys. Rev. D 77, 094502 (2008).

[24] G. S. Bali et al. (QCDSF Collaboration), Strangeness Contribution to the Proton Spin from Lattice QCD, Phys. Rev. Lett. 108, 222001 (2012).

[25] Y. B. Yang, R. S. Sufian, A. Alexandru, T. Draper, M. J. Glatzmaier, K. F. Liu, and Y. Zhao, Glue Spin and Helicity in the Proton from Lattice QCD, Phys. Rev. Lett. 118, 102001 (2017).

[26] C. Alexandrou, M. Constantinou, K. Hadjiyiannakou, K. Jansen, C. Kallidonis, G. Koutsou, A. Vaquero Avils-Casco, and C. Wiese, Nucleon Spin and Momentum Decomposition Using Lattice QCD Simulations, Phys. Rev. Lett. 119, 142002 (2017). 
[27] N. Yamanaka et al. (JLQCD Collaboration), Nucleon charges with dynamical overlap fermions, Phys. Rev. D 98, 054516 (2018).

[28] F. Myhrer and A. W. Thomas, A possible resolution of the proton spin problem, Phys. Lett. B 663, 302 (2008).

[29] A. W. Thomas, Interplay of Spin and Orbital Angular Momentum in the proton, Phys. Rev. Lett. 101, 102003 (2008).

[30] B. S. Zou and D. O. Riska, $s \bar{s}$ Component of the Proton and the Strangeness Magnetic Moment, Phys. Rev. Lett. 95, 072001 (2005).

[31] C.S. An, D. O. Riska, and B.S. Zou, Strangeness spin, magnetic moment and strangeness configurations of the proton, Phys. Rev. C 73, 035207 (2006).

[32] C. Adamuscin, E. Tomasi-Gustafsson, E. Santopinto, and R. Bijker, Two-component model for the axial form factor of the nucleon, Phys. Rev. C 78, 035201 (2008).

[33] R. Bijker, J. Ferretti, and E. Santopinto, $s \bar{s}$ sea pair contribution to electromagnetic observables of the proton in the unquenched quark model, Phys. Rev. C 85, 035204 (2012).

[34] C. S. An and B. S. Zou, The role of the qqqq $\bar{q}$ components in the electromagnetic transition $\gamma * \mathrm{~N} \rightarrow \mathrm{N}(1535)$, Eur. Phys. J. A 39, 195 (2009).

[35] C. An and B. Saghai, Strong decay of low-lying $S_{11}$ and $D_{13}$ nucleon resonances to pseudoscalar mesons and octet baryons, Phys. Rev. C 84, 045204 (2011).

[36] Q. B. Li and D. O. Riska, Five-quark components in $\Delta(1232) \rightarrow \mathrm{N} \pi$ decay, Phys. Rev. C 73, 035201 (2006).

[37] Q. B. Li and D. O. Riska, The role of $q \bar{q}$ components in the N(1440) resonance, Phys. Rev. C 74, 015202 (2006).
[38] C. S. An and B. Saghai, Sea flavor content of octet baryons and intrinsic five-quark Fock states, Phys. Rev. C 85, 055203 (2012).

[39] C. S. An and B. Saghai, Orbital angular momentum of the proton and intrinsic five-quark Fock states, Phys. Rev. D 99, 094039 (2019).

[40] L. Y. Glozman and D. O. Riska, The spectrum of the nucleons and the strange hyperons and chiral dynamics, Phys. Rep. 268, 263 (1996).

[41] M. Mestayer et al. (CLAS Collaboration), Strangeness Suppression of $q \bar{q}$ Creation Observed in Exclusive Reactions, Phys. Rev. Lett. 113, 152004 (2014).

[42] W. C. Chang and J.C. Peng, Flavor Asymmetry of the Nucleon Sea and the Five-Quark Components of the Nucleons, Phys. Rev. Lett. 106, 252002 (2011).

[43] J. Green et al., Up, down, and strange nucleon axial form factors from lattice QCD, Phys. Rev. D 95, 114502 (2017).

[44] C. Alexandrou et al., Complete flavor decomposition of the spin and momentum fraction of the proton using lattice QCD simulations at physical pion mass, Phys. Rev. D 101, 094513 (2020).

[45] S. D. Bass and A. W. Thomas, The Nucleon's octet axialcharge $g_{A}^{(8)}$ with chiral corrections, Phys. Lett. B 684, 216 (2010).

[46] H. Li, P. Wang, D. B. Leinweber, and A. W. Thomas, Spin of the proton in chiral effective field theory, Phys. Rev. C 93, 045203 (2016).

[47] X. G. Wang, C. R. Ji, W. Melnitchouk, Y. Salamu, A. W. Thomas, and P. Wang, Strange quark helicity in the proton from chiral effective theory, Phys. Rev. D 102, 116020 (2020). 